$\underline{\mathbf{P}-38}$

\title{
Small Peptide Molecules for Therapeutics
}

\author{
Narendra Babu SN*
}

School of Pharmacy, Taylor's University, Taylor's University Lakeside Campus, No 1, Jalan Taylor's, Subang Jaya, Selangor, 47500, Malaysia

Peptides are becoming more of interest in the area of drug discovery and delivery [1]. Some peptides have already reached the global market sales. Their different mechanism of action [3] compared to the conventional antibiotics made them prominent leads for searching novel molecules against resistant pathogens. More drug discovery companies are interested in peptides as therapeutic lead molecules. Solving the problems of peptides for their short half-life and their delivery problems is the current challenge. In this context, we designed some small peptides as antibacterial, antitubercular and HIV-1 protease enzyme inhibitors. The synthesis of these small peptides achieved by solution phase peptide synthesis, their structural characterisation and their therapeutic activities are discussed.

\section{ACKNOWLEDGEMENTS}

Author wish to thank School of Pharmacy, Taylor's University, Subang Jaya, Selangor, Malaysia for the research facilities.

\section{REFERENCES}

